\title{
Arterial Stiffness Predicts Cognitive Decline in Japanese Community- dwelling Elderly Subjects: A One-year Follow-up Study
} \author{
Daiki Adachi ${ }^{1}$, Takayuki Hotta ${ }^{1}$, Saori Morino ${ }^{1}$, Yuto Tashiro ${ }^{1}$, Tomoki Aoyama ${ }^{1}$ and Hidenori Arai ${ }^{1,4}$ \\ ${ }^{1}$ Department of Human Health Sciences, Kyoto University Graduate School of Medicine, Kyoto, Japan \\ ${ }^{2}$ Graduate School of Comprehensive Human Sciences, University of Tsukuba, Tokyo, Japan \\ ${ }^{3}$ Japan Society for the Promotion of Science, Tokyo, Japan \\ ${ }^{4}$ National Center for Geriatrics and Gerontology, Obu, Japan
}

Taiki Yukutake ${ }^{1}$, Minoru Yamada ${ }^{1,2}$, Naoto Fukutani ${ }^{1}$, Shu Nishiguchi ${ }^{1,3}$, Hiroki Kayama ${ }^{1}$, Takanori Tanigawa ${ }^{1}$,

\begin{abstract}
Aim: The purpose of this study was to determine whether arterial stiffness can be used to predict one-year changes in the cognitive function in Japanese community-dwelling elderly subjects.

Methods: A total of 103 Japanese community-dwelling elderly patients joined this study. Information regarding the age, height, weight, gender and past medical history of each participant was obtained. Additionally, arterial stiffness was determined according to the cardio-ankle vascular index (CAVI), and the cognitive function was assessed with the Mini-Mental State Examination (MMSE). One year later, we performed the MMSE in the same subjects. After dividing the cohort according to the 80th percentile of the CAVI (normal and arterial stiffness [AS] groups), we examined whether the degree of cognitive decline, as determined using the pre- and post-MMSE, was significantly different based on the severity of arterial stiffness, adjusted for age, BMI, gender and the pre-MMSE scores.

Results: Of the 103 subjects who participated in the pre-data collection, 74 (38 men and 36 women, $73.4 \pm 4.0$ years) joined the post-data collection. We found a significant difference in the change in the post-MMSE scores between the normal and AS groups (pre-MMSE: normal group [27.4 \pm 2.1$]$ and AS group [26.9 \pm 2.4 ] and post-MMSE: normal group [27.2 \pm 2.1$]$ and AS group [25.5 \pm 2.3 ], $F=5.95, p=0.02)$. For each domain of the MMSE, the changes in MMSE-attention-and-calculation $(F=5.11, p=0.03)$ and MMSE-language $(F=4.32, p=0.04)$ were significantly different according to an ANCOVA.

Conclusions: We found that arterial stiffness predicts cognitive decline in Japanese community-dwelling elderly subjects regardless of the initial level of the global cognitive function. This finding indicates the potential use of the degree of arterial stiffness as an indicator for preventing or delaying the onset of dementia in the elderly.
\end{abstract}

J Atheroscler Thromb, 2015; 22: 637-644.

Key words: Arterial stiffness, Cognitive impairment, Elderly, Dementia

\section{Introduction}

Dementia is a serious issue, especially in community-dwelling elderly subjects ${ }^{1}$. Thirty-five million people worldwide suffered from dementia in 2012

Address for correspondence: Hidenori Arai, National Center for Geriatrics and Gerontology, 7-430 Morioka-cho, Obu, Aichi 474-8511, Japan

E-mail: harai@ncgg.go.jp

Received: July 31, 2014

Accepted for publication: October 31, 2014 according to the World Health Organization. Approximately $48 \%$ of patients with Alzheimer's disease (AD), the most common form of dementia, are estimated to live in Asia, and this percentage is expected to increase to $59 \%$ by $2050^{2)}$. Elderly people with dementia are typically frail due to their poor mobility and body composition, and the transitional stage between normal aging and $\mathrm{AD}$, called mild cognitive impairment (MCI), results in frailty ${ }^{3)}$, depression ${ }^{4)}$, lower levels of physical activity ${ }^{1)}$ and higher mortality $^{5)}$. Preventing cognitive decline is therefore crucial. 
Of risk factors for cognitive decline, cardiovascular risk factors have received more attention in recent years ${ }^{6,7)}$. High blood pressure ${ }^{8)}$, dyslipidemia ${ }^{8)}$, obesity $^{9)}$ and diabetes mellitus ${ }^{9)}$ have been proposed to be risk factors for cognitive decline. Among these factors, arterial stiffness is a comparatively easy-to-modify risk factor in community-dwelling elderly subjects. Madden et al. reported that three months of aerobic training reduces the degree of multifactorial arterial stiffness without generating any significant improvements in aerobic fitness, weight, BMI, waist-to-hip ratio or blood pressure in community-dwelling older individu$\mathrm{als}{ }^{10)}$. Additionally, previous studies have demonstrated the effectiveness of antihypertensive agents in improving arterial stiffness in both short- and longterm trials ${ }^{11)}$. Community-dwelling elderly can improve their arterial stiffness; therefore, focusing on treating arterial stiffness may be effective for preventing cognitive decline.

Most older adults with MCI live in the community, and more than half of MCI cases progress to dementia within five years ${ }^{12)}$. Therefore, a desired goal is the early detection of cognitive decline, especially in the community-dwelling elderly. When evaluating the degree of arterial stiffness in community-dwelling elderly subjects, the most important property is the ease of measurement. Arterial stiffness is one of the most easily measured vascular risk factors in community-dwelling elderly patients due to its non-invasive nature; therefore, it can be used as a predictor of cognitive decline in this population. Previous studies have also shown arterial stiffness to be a predictor of cognitive decline. However, the subjects in these studies were not elderly individuals living in the community $\left.{ }^{13},{ }^{14}\right)$. Additionally, other authors have reported that they were unable to validate arterial stiffness as an independent risk factor for cognitive decline, as measured according to the global cognitive function using the Mini-Mental State Examination (MMSE) ${ }^{15-17)}$. Yamamoto et al. reported a relationship between the cognitive function and arterial stiffness determined according to the CAVI in community dwelling elderly ${ }^{18)}$, although the mean age was approximately 80 years, which is a bit high considering the mean age of community-dwelling elderly individuals in Japan. It may be more important to focus on healthier and younger older adults when discussing communitydwelling elderly ${ }^{19)}$. The efficacy of arterial stiffness as a predictor of cognitive decline, especially in community-dwelling elderly patients, is less well investigated $^{4)}$.

The purpose of this study therefore was to address whether the degree of arterial stiffness can be used to predict one-year changes in the cognitive function in Japanese community-dwelling elderly subjects. We used the CAVI to assess arterial stiffness, as this parameter was found to significantly correlate with cognitive decline in a cross-sectional study ${ }^{18,19)}$.

\section{Methods}

\section{Participants}

Participants were recruited for this study through local press that requested healthy community-dwelling volunteers 65 years of age or older, and data collection was performed on two occasions: November 2012 (pre-data collection) and November 2013 (post-data collection). Interviews were conducted to exclude participants from both data collections based on the following exclusion criteria: severe cardiac, pulmonary or musculoskeletal disorders; comorbidities associated with a higher risk of falls, such as Parkinson's disease or stroke; and the use of psychotropic drugs. Written informed consent was obtained from each participant in accordance with the guidelines approved by the Kyoto University Graduate School of Medicine and the Declaration of Human Rights, Helsinki, 1995 during both data collection periods. The study protocol was approved by the ethics committee of Kyoto University Graduate School of Medicine.

\section{Measurements - Pre-data Collection Demographic Data}

Each patient's age, height, weight, gender, past medical history (cardiovascular disease, hypertension, diabetes mellitus and hyperlipidemia), smoking status (number of cigarettes smoked per day and total number of years smoked) and educational background (elementary school, junior high school, high school, career college or university) were obtained as demographic data. All data were collected at the first data collection time point. We directly asked about each participant's age and gender and measured their height and weight using standardized height and weight scales.

\section{Arterial Stiffness}

The degree of arterial stiffness was determined based on the CAVI using the VaSera-1500 device (Fukuda Denshi Co., Ltd., Tokyo, Japan). The details of this procedure have been described previously ${ }^{20,21)}$. After the participants had rested for five minutes in the sitting position, we obtained these measurements as previously described. Higher CAVI values indicate a higher degree of arterial stiffness. The measurements were obtained once, and the mean values of the right 
Table 1. Baseline characteristics and post-MMSE scores in the study population

\begin{tabular}{|c|c|c|c|}
\hline & \multicolumn{3}{|c|}{ All $(n=74)$} \\
\hline & $\begin{array}{c}\text { Normal group } \\
n=59\end{array}$ & $\begin{array}{c}\text { AS group } \\
n=15\end{array}$ & $p$ \\
\hline \multicolumn{4}{|l|}{ Demographic data } \\
\hline Age, year & $72.8 \pm 3.8$ & $76.1 \pm 3.6$ & $<0.01$ \\
\hline BMI, kg/m² & $23.2 \pm 2.6$ & $23.2 \pm 3.2$ & 0.99 \\
\hline Gender, male & $28(47.5 \%)$ & $10(66.7 \%)$ & 0.25 \\
\hline Mean CAVI & $8.83 \pm 0.61$ & $10.6 \pm 0.51$ & $<0.01$ \\
\hline \multicolumn{4}{|l|}{ Cognitive function } \\
\hline Pre-MMSE & $27.4 \pm 2.1$ & $26.9 \pm 2.4$ & 0.40 \\
\hline Post-MMSE & $27.2 \pm 2.0$ & $25.5 \pm 2.3$ & $<0.01$ \\
\hline Pre-MMSE (orientation) & $9.6 \pm 0.6$ & $9.7 \pm 0.5$ & 0.89 \\
\hline Post-MMSE (orientation) & $9.7 \pm 0.7$ & $9.7 \pm 0.5$ & 0.89 \\
\hline Pre-MMSE (registration) & $2.9 \pm 0.4$ & $3.0 \pm 0.0$ & 0.53 \\
\hline Post-MMSE (registration) & $2.9 \pm 0.3$ & $3.0 \pm 0.0$ & 0.49 \\
\hline Pre-MMSE (attention and calculation) & $3.2 \pm 1.7$ & $2.9 \pm 1.8$ & 0.55 \\
\hline Post-MMSE (attention and calculation) & $3.4 \pm 1.7$ & $2.3 \pm 1.5$ & 0.03 \\
\hline Pre-MMSE (recall) & $2.6 \pm 0.6$ & $2.4 \pm 0.8$ & 0.30 \\
\hline Post-MMSE (recall) & $2.5 \pm 0.6$ & $2.4 \pm 0.7$ & 0.69 \\
\hline Pre-MMSE (language) & $8.9 \pm 0.3$ & $8.9 \pm 0.4$ & 0.73 \\
\hline Post-MMSE (language) & $8.7 \pm 0.5$ & $8.2 \pm 1.3$ & 0.15 \\
\hline \multicolumn{4}{|l|}{ Comorbidities } \\
\hline Cardiovascular disease & $6(10.2 \%)$ & $4(26.7 \%)$ & 0.11 \\
\hline Hypertension & $23(39.0 \%)$ & $8(53.3 \%)$ & 0.39 \\
\hline Diabetes mellitus & $5(8.5 \%)$ & $4(26.7 \%)$ & 0.08 \\
\hline Hyperlipidemia & $9(15.3 \%)$ & $2(13.3 \%)$ & 1.00 \\
\hline Brinkman index & $0(0-800)$ & $0(0-400)$ & 0.63 \\
\hline Educational background & & & n.s. \\
\hline Elementary school & $0(0.0 \%)$ & $1(6.7 \%)$ & \\
\hline Junior high school & $16(27.1 \%)$ & $4(26.7 \%)$ & \\
\hline High school & $35(59.3 \%)$ & $9(60.0 \%)$ & \\
\hline Career college & $3(5.1 \%)$ & $0(0.0 \%)$ & \\
\hline University & $5(8.5 \%)$ & $1(6.7 \%)$ & \\
\hline
\end{tabular}

Mean CAVI $=$ mean value of the right and left CAVI scores. The mean \pm SD is shown for age, BMI, mean CAVI and pre- and post MMSE. $\mathrm{n}(\%)$ is shown for gender, cardiovascular disease, hypertension, diabetes mellitus, hyperlipidemia and educational background. The median (25\% quartile-75\% quartile) is shown for the Brinkman index. AS: arterial stiffness; n.s.: not significant.

and left CAVI scores for each patient were used for the analysis ${ }^{19)}$.

\section{Cognitive Function Measurements}

The cognitive function was assessed using the Mini-Mental State Examination (MMSE) ${ }^{22)}$. The MMSE is a short screening test that consists of the following five areas for detecting cognitive impairment: orientation, registration, attention and calculation, recall and language. The scores range from 0 to 30 , with higher scores indicating better cognitive performance. The MMSE was performed at both the pre- and post-data collection time points.

\section{Measurements - Post-data Collection Cognitive Function Measurements}

One year later, the cognitive function was also assessed using the MMSE ${ }^{22}$. We performed the MMSE using the same inclusion and exclusion criteria as that used at the pre-data collection time point.

\section{Statistical Analysis}

The patients were divided into two groups based on the 80th percentile of the CAVI values: the normal 


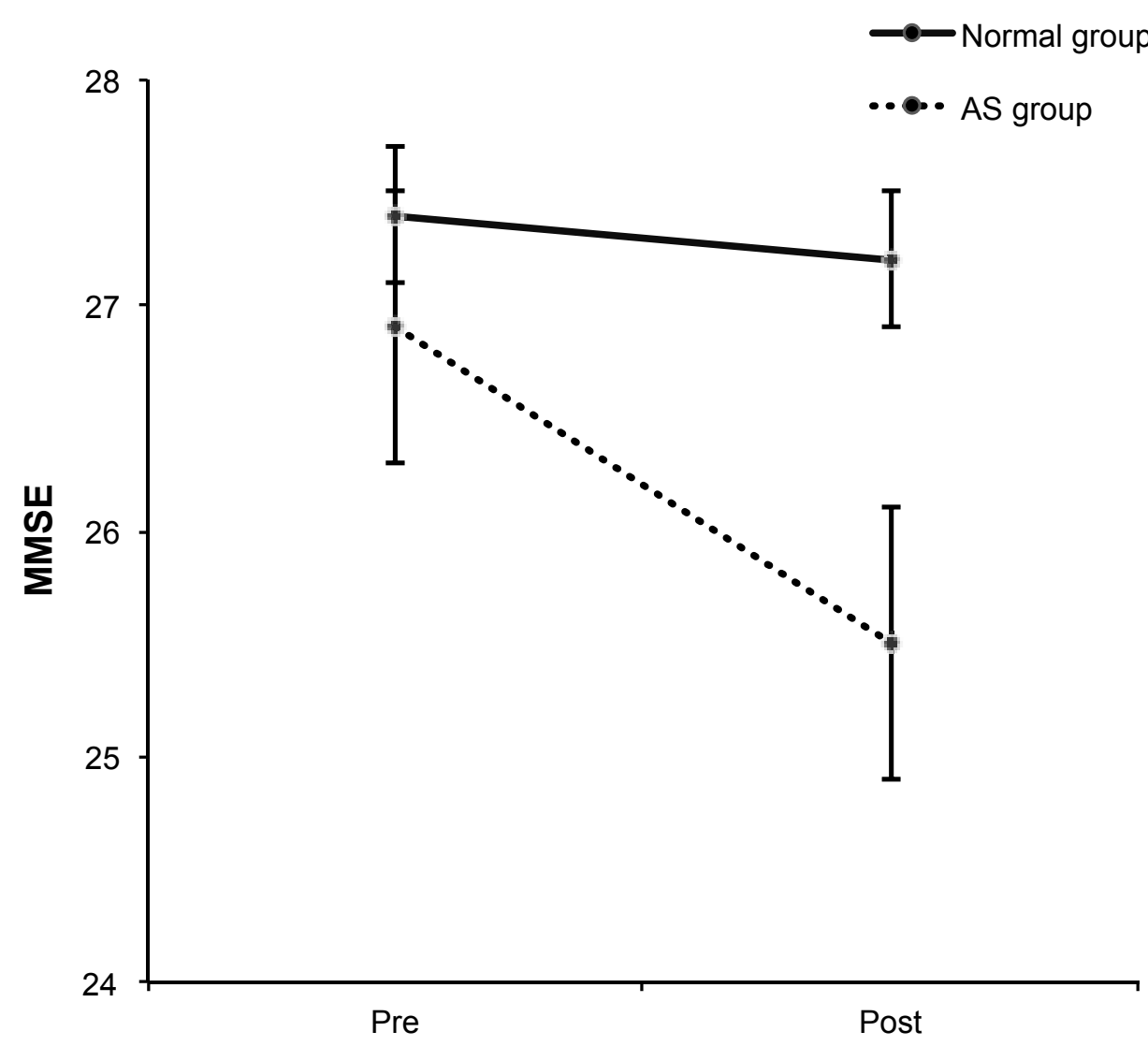

Fig. 1. Two-way analysis of variance showing the differences in the changes in the postMMSE scores between the normal and AS groups. These findings indicate that the elderly subjects in the AS group experienced greater cognitive decline than those in the control group $(F=5.95, p=0.02)$.

and arterial stiffness [AS] groups. We analyzed the differences between these two groups using the unpaired $t$-test for age, body mass index (BMI), mean CAVI values on both sides and the pre- and post-MMSE scores (total score and scores for each domain), the $\chi^{2}$ test for gender, past medical history and educational background and the Mann Whitney $U$-test for the Brinkman index (number of cigarettes smoked per day $x$ total number of years smoked). A repeated measures two-way analysis of covariance (ANCOVA) was used to analyze whether the degree of cognitive decline determined according to the pre- and post-MMSE scores (total score and scores for each domain) differed significantly according to the severity of arterial stiffness, adjusted for age, BMI, gender and the preMMSE score. A $p$ value of $<0.05$ was considered to be statistically significant for all analyses.

\section{Results}

In total, 74 individuals (38 men and 36 women, $73.4 \pm 4.0$ years) participated in both data collection events. Of these individuals, none were excluded. We assigned 59 elderly individuals $(28 \mathrm{men}$ and 31 women) to the normal group and 15 (10 men and five women) to the AS group. Table 1 shows the differences in each variable between the two groups. While there were no significant differences in BMI, gender, pre-MMSE, educational background or past medical history, we found significant differences in age $(p<$ $0.01)$ and the mean CAVI values $(p<0.01)$. Additionally, the normal group had a significantly higher total post-MMSE scores (normal group: 27.2 \pm 2.1 , AS group: $25.5 \pm 2.3, p<0.01)$ and higher post-MMSE scores for the attention-and-calculation domain (normal group: $3.4 \pm 1.7$, AS group: $2.3 \pm 1.5, p=0.03$ ) than the AS group.

The ANCOVA adjusted for age, BMI, gender 


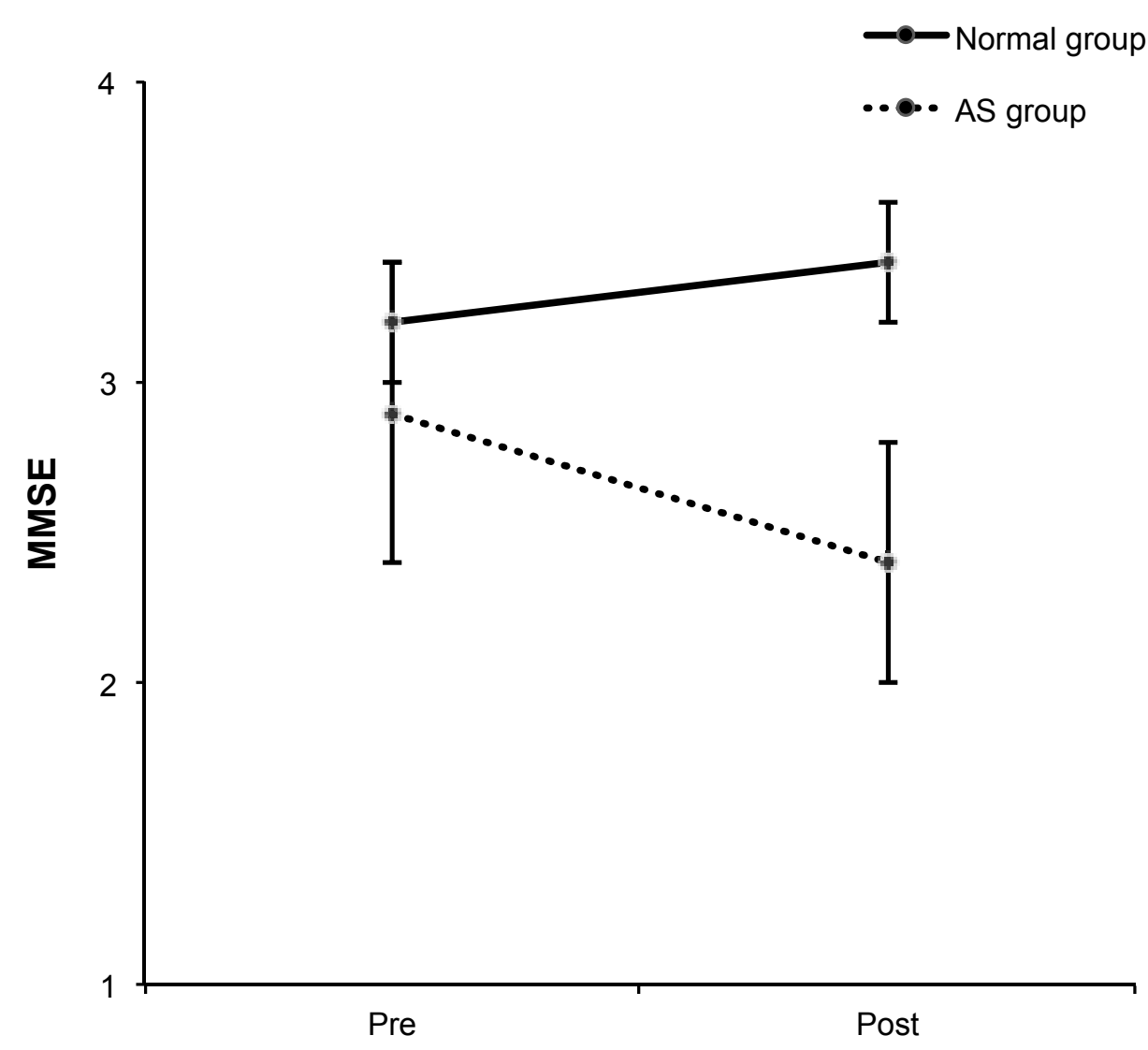

Fig. 2. Two-way analysis of variance showing the differences in the changes in the postMMSE (attention and calculation) scores between the normal and AS groups. These findings indicate that the elderly subjects in the AS group experienced greater cognitive decline than those in the control group $(F=5.11, p=0.03)$.

and pre-MMSE showed a significant difference in the changes in the post-MMSE scores between the normal and AS groups $(F=5.95, p=0.02)$ (Fig. 1), indicating that elderly individuals with a higher degree of arterial stiffness may experience greater levels of cognitive decline, even after adjusting for age, BMI, gender and the pre-MMSE score. Additionally, the changes in the MMSE-attention-and-calculation $(F=5.11, p=0.03)$ (Fig. 2) and MMSE-language $(F=4.32, p=0.04)$ (Fig.3) domains were shown to be significantly different according to the ANCOVA. The other areas did not show any differences between the two groups (orientation; $F=0.27, p=0.60$ : registration; $F=2.69$, $p=$ 0.11 , recall; $F=0.16, p=0.69)$.

\section{Discussion}

In this study, we analyzed whether the degree of cognitive decline differs significantly according to the severity of arterial stiffness, adjusted for age, BMI, gender and the cognitive function at baseline and at the one-year follow-up. Consequently, we found that arterial stiffness predicts cognitive decline in Japanese community-dwelling elderly subjects, regardless of the initial level of the global cognitive function. Previous studies have demonstrated that arterial stiffness has a predictive effect on cognitive decline in the non-community-dwelling elderly ${ }^{13-15,18)}$; however, few reports have found arterial stiffness to be a predictor of cognitive decline in this group.

There are hypotheses regarding pathways linking arterial stiffness and cognitive decline, wherein augmented pressure pulses penetrate and damage small cerebral vessels in the global brain ${ }^{23)}$. Brain lesions, such as ischemic lesions and white matter abnormalities resulting from augmented pressure, are thought to cause cognitive decline, thereby leading to dementia $^{24)}$. The augmented pressure caused by arterial stiffness independently predicts cognitive performance ${ }^{25)}$, and many previously published studies evaluating the 


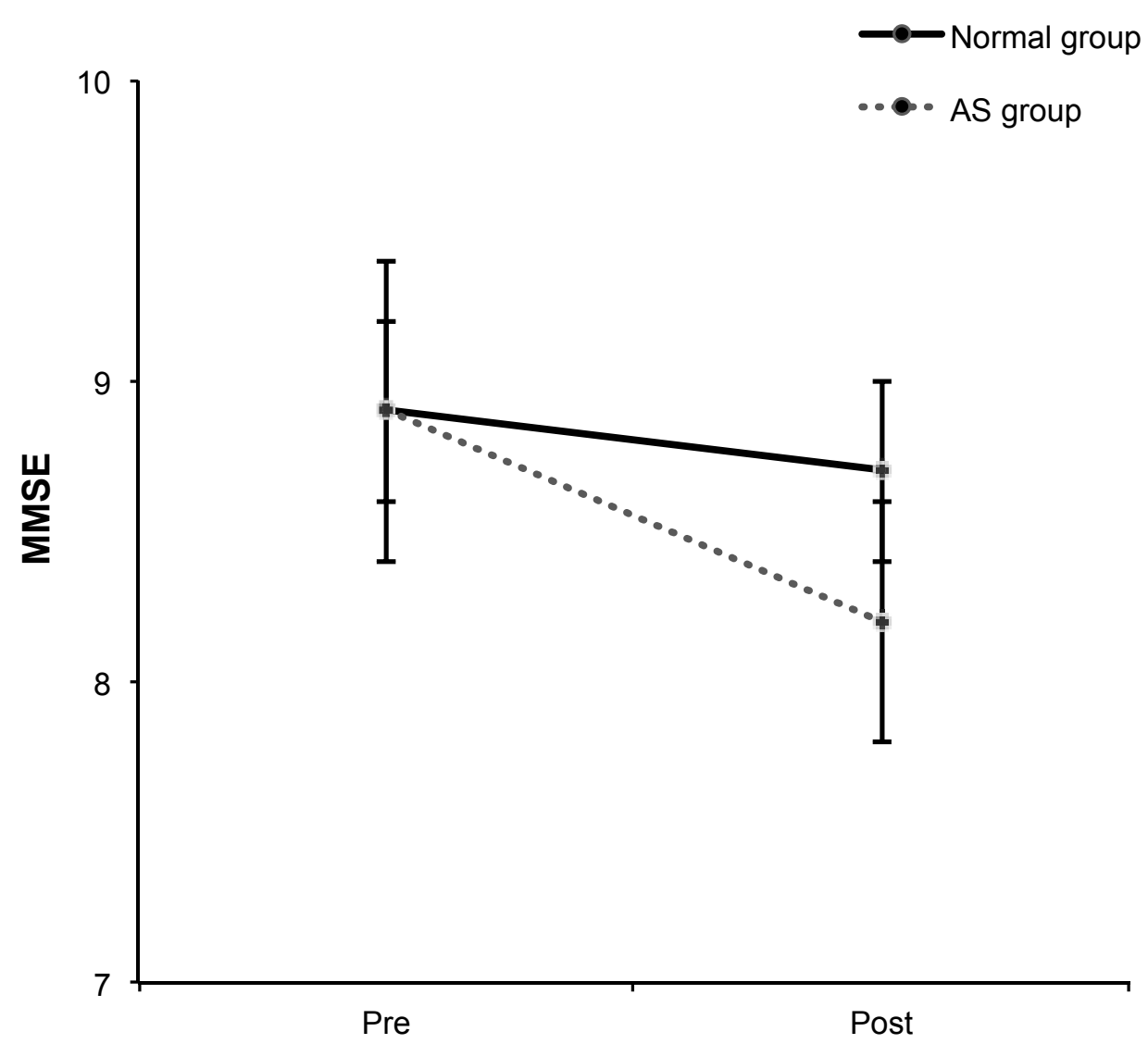

Fig. 3. Two-way analysis of variance showing the differences in the changes in the postMMSE (language) scores between the normal and AS groups. These findings indicate that the elderly subjects in the AS group experienced greater cognitive decline than those in the control group $(F=4.32, p=0.04)$.

association between arterial stiffness and the cognitive function have discussed the causal relationship with this phenomenon ${ }^{14,17,18,23)}$.

Several studies have examined whether the severity of arterial stiffness longitudinally predicts cognitive decline. For example, one study targeting people older than 80 years of age in nursing homes showed results similar to the current findings ${ }^{13)}$. The mean baseline MMSE score of these subjects was $23.7 \pm 4$.9, which is lower than that observed in the current study. Another study, in which the subjects were older patients in the hospital with complaints of memory loss, also reported that arterial stiffness has a strong predictive ability for cognitive decline ${ }^{14)}$. Furthermore, Yamamoto et al. performed a similar analysis in community-dwelling elderly patients; however, the mean age was higher than that noted in our study ${ }^{18)}$. Notably, we found that arterial stiffness predicts cognitive decline in community-dwelling elderly subjects with a comparably preserved cognitive function, even after adjusting for age, gender, BMI and the baseline cognitive function. In addition, we observed the scores for the attentionand-calculation and language domains of the MMSE to be significantly decreased in the AS group. It has been reported that these MMSE domains are not affected by impairment of the hippocampus ${ }^{26)}$. Therefore, we assume that the cognitive dysfunction resulting from arterial stiffness is not attributed to dysfunction of the hippocampus. However, other studies have reported that measurements of arterial stiffness do not predict performance for the global cognitive function, as measured according to the MMSE ${ }^{15-17)}$. There are various possible reasons for this discrepancy: 1) the mean age of the subjects was 57.1 years and the participants were relatively high functioning (ceiling effect of the MMSE) ${ }^{15)}$; 2) many participants dropped out from the follow-up survey and selection bias may have affected the results for the change in the cognitive function ${ }^{16)}$; 3) memory tasks that are more demanding for the executive function and attention 
may be more sensitive to cerebrovascular alterations due to aging and the MMSE may be too insensitive to accurately detect cognitive changes ${ }^{17,27)}$. As a result, further studies are needed to establish evidence clarifying the association between arterial stiffness and the cognitive function.

The most important clinical implication of our findings is that one of the most easily measured and non-invasive parameters, especially in communitydwelling elderly individuals, arterial stiffness, predicted cognitive decline after one year. These results imply that maintaining the arterial function may prevent or delay the onset of dementia in the community-dwelling elderly. Additionally, it may be possible to identify individuals at risk of dementia by evaluating the degree of arterial stiffness. Interventional and longitudinal studies examining improvements in arterial stiffness with the aim of preventing cognitive decline are required to establish effective strategies for inhibiting the onset of dementia.

This study is associated with several limitations. First, because we were unable to perform neuroimaging assessments, it was not possible to make a specific diagnosis of dementia subtypes. In addition, we only performed MMSE as a cognitive test, and the cognitive function was not fully investigated. There may be asymptomatic brain lesions and specific cognitive domains that exhibit a strong relationship with arterial stiffness. Second, the age at baseline in the AS group was significantly higher than that observed in the normal group. Although we tried to minimize the impact of this difference by adjusting for age, the effect may have been insufficient. Third, the small number of subjects may also have affected the results, and more samples are needed to confirm the results of this study. Finally, many studies have investigated the relationship between arterial stiffness and the cognitive function; therefore, this study may not have adequate novelty. Nevertheless, we regard our findings as providing evidence that strengthens the close relationship between arterial stiffness and cognitive decline.

\section{Conclusions}

This study showed that arterial stiffness predicts cognitive decline in Japanese community-dwelling elderly subjects regardless of the initial level of the global cognitive function. These findings indicate the potential of improving arterial stiffness in order to prevent or delay of the onset of dementia in the elderly.

\section{Acknowledgments}

This work was supported in part by Grants-inAid for Comprehensive Research on Aging and Health from the Ministry of Health, Labor, and Welfare, Japan (H24-Tyojyu-001).

\section{Conflicts of Interest}

None.

\section{References}

1) Weuve J, Kang JH, Manson JE, Breteler MM, Ware JH, Grodstein F: Physical activity, including walking, and cognitive function in older women. JAMA, 2004; 292: 1454-1461

2) Brookmeyer R, Johnson E, Ziegler-Graham K, Arrighi HM: Forecasting the global burden of Alzheimer's disease. Alzheimers Dement, 2007; 3: 186-191

3) Kulmala J, Nykänen I, Mänty M, Hartikainen S: Association between Frailty and Dementia: A Population-Based Study. Gerontology, 2014; 60: 16-21

4) Solfrizzi V, D'Introno A, Colacicco AM, Capurso C, Del Parigi A, Caselli RJ, Scapicchio PL, Scafato E, Gandin C, Capurso A, Panza F: Incident occurrence of depressive symptoms among patients with mild cognitive impairment - the Italian longitudinal study on aging. Dement Geriatr Cogn Disord, 2007; 24: 55-64

5) Tehrani DM, Darki L, Erande A, Malik S: In-hospital mortality and coronary procedure use for individuals with dementia with acute myocardial infarction in the United States. J Am Geriatr Soc, 2013; 61: 1932-1936

6) Arntzen KA, Schirmer H, Wilsgaard T, Mathiesen EB: Impact of cardiovascular risk factors on cognitive function: the Tromsø study. Eur J Neurol, 2011; 18: 737-743

7) Stephan BC, Brayne C: Vascular factors and prevention of dementia: Int Rev Phychiatry, 2008; 20: 344-356

8) Okusaga O, Stewart MCW, Butcher I, Deary I, Fowkes FG, Price JF: Smoking, hypercholesterolaemia and hypertension as risk factors for cognitive impairment in older adults. Age Ageing, 2013; 42: 306-311

9) Ott A, Stolk RP, van Harskamp F, Pols HA, Hofman A, Breteler MM: Diabetes mellitus and the risk of dementia: the Rotterdam study. Neurology, 1999; 53: 1937-1942

10) Madden KM, Lockhart C, Cuff D, Potter TF, Meneilly GS: Short-term aerobic exercise reduces arterial stiffness in older adults with type 2 diabetes, hypertension, and hypercholesterolemia. Diabetes Care, 2009; 32: 15311535

11) Asmar R: Effect of antihypertensive agents on arterial stiffness as evaluated by pulse wave velocity: clinical implications. Am J Cardiovasc Drugs, 2001; 1: 387-397

12) Gauthier S, Reisberg B, Zaudig M, Petersen RC, Ritchie $\mathrm{K}$, Broich K, Belleville S, Brodaty H, Bennett D, Chertkow $\mathrm{H}$, Cummings JL, de Leon M, Feldman $\mathrm{H}$, Ganguli M, Hampel H, Scheltens P, Tierney MC, Whitehouse P, Winblad B: Mild cognitive impairment. Lancet, 2006; 
367: 1262-1270

13) Benetos A, Watfa G, Hanon O, Salvi P, Fantin F, Toulza O, Manckoundia P, Agnoletti D, Labat C, Gautier S; PARTAGE Study Investigators: Pulse wave velocity is associated with 1-year cognitive decline in the elderly older than 80 years: the PARTAGE study. J Am Med Dir Assoc, 2012; 13: 239-243

14) Scuteri A, Tesauro M, Appolloni S, Preziosi F, Brancati AM, Volpe M: Arterial stiffness as an independent predictor of longitudinal changes in cognitive function in the older individual. J Hypertens, 2007; 25: 1035-1040

15) Waldstein SR, Rice SC, Thayer JF, Najjar SS, Scuteri A, Zonderman AB: Pulse pressure and pulse wave velocity are related to cognitive decline in the Baltimore Longitudinal Study of Aging. Hypertension, 2008; 51: 99-104

16) Poels MM, van Oijen M, Mattace-Raso FU, Hofman A, Koudstaal PJ, Witteman JC, Breteler MM: Arterial stiffness, cognitive decline, and risk of dementia: the Rotterdam study. Stroke, 2007; 38: 888-892

17) Watson NL, Sutton-Tyrrell K, Rosano C, Boudreau RM, Hardy SE, Simonsick EM, Najjar SS, Launer LJ, Yaffe K, Atkinson HH, Satterfield S, Newman AB: Arterial stiffness and cognitive decline in well-functioning older adults. J Gerontol A Biol Sci Med Sci, 2011; 66: 13361342

18) Yamamoto N, Yamanaka G, Ishikawa M, Takasugi E, Murakami S, Yamanaka T, Ishine M, Matsubayashi K, Hanafusa T, Otsuka K: Cardio-ankle vascular index as a predictor of cognitive impairment in community-dwelling elderly people: four-year follow-up. Dement Geriatr Cogn Disord, 2009; 28: 153-158

19) Yukutake T, Yamada $M$, Fukutani $N$, Nishiguchi $S$, Kayama H, Tanigawa T, Adachi D, Hotta T, Morino S, Tashiro Y, Arai H, Aoyama T: Arterial Stiffness Determined According to the Cardio-Ankle Vascular Index
(CAVI) is Associated with Mild Cognitive Decline in Community-Dwelling Elderly Subjects: J Atheroscler Thromb, 2014; 21: 49-55

20) Shirai K, Hiruta N, Song M, Kurosu T, Suzuki J, Tomaru T, Miyashita Y, Saiki A, Takahashi M, Suzuki K, Takata M: Cardio-Ankle Vascular Index (CAVI) as a novel indicator of arterial stiffness: theory, evidence, and perspectives. J Atheroscler Thromb, 2011; 18: 924-938

21) Shirai K, Utino J, Otsuka K, Takata M: A novel blood pressure-independent arterial wall stiffness parameter; Cardio-Ankle Vascular Index (CAVI). J Atheroscler Thromb, 2006; 13: 101-107

22) Folstein MF, Folstein SE, McHugh PR: "Mini-mental state". A practical method for grading the cognitive state of patients for the clinician: J Psychiatr Res, 1975; 12: 189-198

23) Pase MP, Herbert A, Grima NA, Pipingas A, O'Rourke MF: Arterial stiffness as a cause of cognitive decline and dementia: a systematic review and meta-analysis. Intern Med J, 2012; 42: 808-815

24) Pasquier F, Leys D: Why are stroke patients prone to develop dementia? J Neurol, 1997; 224: 135-142

25) Pase MP, Pipingas A, Kras M, Nolidin K, Gibbs AL, Wesnes KA, Scholey AB, Stough C: Healthy middle-aged individuals are vulnerable to cognitive deficit as a result of increased arterial stiffness. J Hypertens, 2010; 28: 17241729

26) Shigemori K, Ohgi S, Okuyama E, Shimura T, Schneider E: The factorial structure of the Mini-Mental State Examination (MMSE) in Japanese dementia patients: BMC Geriatr, 2010; 10: 36

27) Buckner RL: Memory and executive function in aging and AD: multiple factors that cause decline and reserve factors that compensate. Neuron, 2004; 44: 195-208 